University of Nebraska - Lincoln

DigitalCommons@University of Nebraska - Lincoln

April 1988

\title{
Translational-rotational modes in noncentrosymmetric lattices: A lattice-dynamical interpretation of the phenanthrene phase transition
}

\author{
R. Kulver \\ University of Nebraska - Lincoln \\ Craig J. Eckhardt \\ University of Nebraska - Lincoln, ceckhardt1@unl.edu
}

Follow this and additional works at: https://digitalcommons.unl.edu/chemistryeckhardt

Part of the Chemistry Commons

Kulver, R. and Eckhardt, Craig J., "Translational-rotational modes in noncentrosymmetric lattices: A latticedynamical interpretation of the phenanthrene phase transition" (1988). Craig J. Eckhardt Publications. 24. https://digitalcommons.unl.edu/chemistryeckhardt/24

This Article is brought to you for free and open access by the Published Research - Department of Chemistry at DigitalCommons@University of Nebraska - Lincoln. It has been accepted for inclusion in Craig J. Eckhardt Publications by an authorized administrator of DigitalCommons@University of Nebraska - Lincoln. 


\title{
Translational-rotational modes in noncentrosymmetric lattices: A lattice-dynamical interpretation of the phenanthrene phase transition
}

\author{
R. Kulver and C. J. Eckhardt \\ Department of Chemistry, University of Nebraska, Lincoln, Nebraska 68588-0304
}

(Received 20 July 1987)

\begin{abstract}
A lattice-dynamical study has been used to elucidate the mechanism of the phase transition in phenanthrene. The calculation is consistent with the known lattice energy and crystal structure. Combination of experimental results with the calculated phonon dispersion curves and eigenmode symmetries yields a dynamic mechanism for the transition based on increasing density of higherfrequency modes upon approach to the transition temperature to the high-temperature phase. This motion, which may be associated with motion of hydrogen atoms in the molecule, confers a more three-dimensional character to the interactions and correlates well with the observed change in heat capacity.
\end{abstract}

\section{INTRODUCTION}

Molecular solids present challenging problems in the understanding of lattice dynamics. These range from the determination of the coupling between internal and external phonon modes to questions concerning phase transitions that cannot be explained by the traditional softmode mechanisms. Indeed, with increased interest in organic materials and solid-state reactions, the understanding of the lattice dynamics of molecular crystals becomes more compelling.

Any such investigation is essentially focused on the molecular dynamics in the solid state and the nature of the intermolecular potentials governing them. For such studies all intermolecular motion and degrees of freedom may influence the dynamical behavior of the crystal.

In the study of the lattice dynamics of molecular crystals, the aromatic hydrocarbons have served as the most common model systems. Of these, anthracene has received a great deal of attention as the archetype. Its rigid, planar, geometry with its concomitant lack of lowfrequency internal modes makes it ideal for the study of the external phonon modes. However, anthracene's centric lattice ${ }^{1}\left(P 2_{1} / a\right)$ prevents study of the question of coupling between translational and librational modes. Further, its use as a model for a molecular crystal phase transition is much more limited with only some experimental evidence of an intergrowth triclinic phase. ${ }^{2,3}$

Phenanthrene, a lower symmetry, structural isomer of anthracene, also packs into a lower-symmetry lattice ${ }^{4}$ $\left(P 2_{1}\right)$ than the latter. The crystallization of a lowsymmetry molecule into a low-symmetry lattice is not uncommon but there exists no reliable criterion for prediction of the crystal structure from molecular geometry. This may be regarded as another central problem in the lattice dynamics of molecular crystals.

The solid-state properties of the two isomers differ significantly and the less efficient packing of the phenanthrene crystal certainly contributes to the discrepancies. Anthracene has a melting point of $216^{\circ} \mathrm{C}$ whilst that of phenanthrene is at $100^{\circ} \mathrm{C}$. The enthalpies of sublimation ${ }^{5}$ show a variance with that of anthracene at $101.0 \mathrm{~kJ} / \mathrm{mol}$ and of phenanthrene at $86.6 \mathrm{~kJ} / \mathrm{mol}$. The most significant difference is that phenanthrene has an apparent phase transition at ambient pressure while anthracene does not.

The phase transition in phenanthrene has been the object of much study since its behavior is unusual. ${ }^{6-8}$ Heat-capacity studies show a transition from $68-72^{\circ} \mathrm{C}$ with a change in heat capacity of $8 \times 10^{-17} \mathrm{erg} / \mathrm{K} .{ }^{9}$ The presence of a phase transition in this temperature region is supported by slight changes in the electrical conductivity, dielectric constant, and thermal conductivity of the crystal. Further structural evidence is given by $\mathrm{x}$-ray and neutron diffraction studies which show no change in space group and only slight changes in lattice constants. However, the change appears to arise from neither an order-disorder nor displacive change within the rigidbody approximation. The transition appears to be associated with the rearrangement of hydrogen atoms of the molecule. This phase transition would seem to lie somewhere between thermal expansion and a higher than second-order transition. The temperature-dependent diffraction studies leave little uncertainty regarding the crystal structures above and below the transition range, but the mechanism of the transition remains unsolved. The main motivation of this investigation is to understand the mechanism for the phenanthrene phase transition through elucidation of the lattice dynamics of this noncentrosymmetric crystal.

The acentric lattice of phenanthrene complicates the assignment of the lattice modes. The frequencies of the external phonons can be calculated by classical lattice dynamics, but the eigenvectors of the dynamical matrix, which represent the molecular motions, become difficult to interpret.

Centrosymmetric lattices exhibit vibrational modes that can be reduced through symmetry to either translational (infrared active) or rotational (Raman active) modes. The acentric lattice of phenanthrene is under no such symmetry constraint, and thus mixing of translational and rotational motions may be expected. In this 
study we examine the geometry of these complex motions directly by obtaining the eigenvectors of tha dynamical matrix following the classical formalism. Phonon dispersion curves are subsequently calculated by solving the dynamical problem for a quasicontinuum in reciprocal space. The eigenvectors are then interpreted as molecular motions. From the above information a mechanism for the phase transition in phenanthrene is constructed.

\section{LATTICE ENERGY MINIMIZATION AND CLASSICAL LATTICE DYNAMICS}

There are numerous empirical and semiempirical potential energy functions used in energy calculations of molecular crystals. The one most often utilized is the modified Buckingham or 6-exp-1 atom-atom potential function of the form

$$
V=A_{i j} \exp \left(-B_{i j} r_{i j}\right)-C_{i j} / r_{i j}^{6}+D_{i j} / r_{i j},
$$

where $r_{i j}$ is the distance between an atom of type $i$ on one molecule and an atom of type $j$ on another molecule. All pairwise interactions are summed over molecules in several unit cells.

In order to assure that a crystal structure is not under strain when the force constants are evaluated, the potential energy of the lattice must be minimized with respect to unit cell axes and molecular orientations. ${ }^{10}$ The atomatom potential, which is a function of all of the coordinates of all of the atoms in each molecule, can be transformed into a function of six variables to describe the size and shape of the lattice $(a, b, c, \alpha, \beta, \gamma)$ and six to describe the position and orientation of each molecule (Euler angles and center-of-mass coordinates). Symmetry considerations can be used to further reduce the number of variables in the energy sum but, in general, it is a 12dimensional hypersurface.

The most reliable test of a crystal potential function is the comparison of the lattice structure at the energy minimum to the experimentally determined $x$-ray structure, ideally at the same temperature. This calculation was performed at $1 \mathrm{~K}$, where lattice energy is essentially equal to the enthalpy of sublimation. Therefore, the $\mathrm{x}$ ray structure taken at the lowest possible temperature is the structure that should best compare to the results of this energy minimization. If the calculated and experimental structures show a favorable agreement, then one can be assured that the lattice is under no strain and that the potential can be expressed as a truncated Taylorseries expansion. Improved carbon-hydrogen parameters calculated by Williams ${ }^{11}$ were used for the $A, B$, and $C$ values, and partial charges were calculated using charge densities obtained by STO/3G molecular orbital calculations. $^{12}$ A minimized lattice energy of $89.37 \mathrm{~kJ} / \mathrm{mol}$ agrees well with the enthalpy of sublimation of phenanthrene of $86.6 \mathrm{~kJ} / \mathrm{mol}$. This favorable agreement shows that a modification of the atom-atom potential need not be made to account for anisotropy effects in the acentric phenanthrene lattice. A three-body potentialenergy term has been added to the Buckingham potential in lattice dynamics calculations by Remizov et al.;13 however, our results show that this modification is un- necessary as the pairwise atom-atom potential yields a lattice energy much closer to the experimental value.

The classical lattice dynamical problem ${ }^{14}$ is set up starting with the equation of motion derived from the constraints of the harmonic approximation:

$$
-m \ddot{u}_{\alpha}(l, k)=\sum_{l^{\prime}, k^{\prime} \beta} \Phi_{\alpha \beta}\left(l, k, l^{\prime}, k^{\prime}\right) u_{\beta}\left(l^{\prime}, k^{\prime}\right) .
$$

Its solution $u_{\alpha}(l, k)$ is the displacement of $k$ th molecule in the $\alpha$ direction due to a movement of the $l^{\prime}, k^{\prime}$ molecule in the $\beta$ direction; $m$ is the mass of one molecule and $\Phi_{\alpha \beta}$ is the force constant with respect to the central molecule. Upon inserting the plarle-wave solution, differentiating with respect to time, and rearranging the force constants into the dynamical matrix, the dynamical problem is reduced to the eigenvalue problem:

$$
\omega(q)^{2} \underline{\mathrm{U}}(q)=\underline{\underline{M}}(q) \underline{\mathbf{U}}(q) .
$$

The eigenvalues of $\underline{\mathcal{M}}(q)$ the dynamical matrix are the quadratic frequencies of the lattice's normal modes, and the corresponding eigenvectors are the six-dimensional vectors composed of three molecular center-of-mass coordinates and three Euler angles describing the orientation of the molecule. These eigenvectors are then directly interpreted as the molecular motions of the optical phonon vibrational modes. The frequencies of the 12 normal modes of vibration, when solved for points in the Brillouin zone from zero to the zone boundary, form a dispersive curve; phonons of different $q$ vector travel in different directions at different velocities through a crystal. The dispersion relation can yield information about the scattering of phonons off other excitations.

\section{CALCULATED PHONON DISPERSION CURVES}

While the zero-wave-vector modes are most often compared to infrared or Raman spectra to determine the accuracy of the potential, the anisotropy of a crystal cannot fully be explored unless the dynamical problem is solved at all points of unique symmetry in the Brillouin zone. The calculated dispersion relation of a crystal is most often compared to those obtained by neutron scattering or Brillouin spectroscopy to determine more precisely the accuracy of the crystal potential in all lattice directions. Calculated dispersion curves for naphthalene and anthracene crystals have been compared to experimental data and have shown reasonably good agreement in all symmetry directions of the reciprocal lattice. ${ }^{15,16}$ Slight modifications to the intermolecular potential, such as addition of charge and multipole interactions, have been introduced to improve the fit of calculated and experimental dispersion curves. While the calculated dispersion relation presented here cannot be compared to any presently available experimental data, information about the anisotropy of the phenanthrene potential can be gleaned from numerous data gathered on its phase transition observed near $345 \mathrm{~K}$. 


\section{SOLID-SOLID PHASE TRANSITION IN PHENANTHRENE}

The x-ray crystal structure of phenanthrene shows that the molecule is forced to be nonplanar by the two hydrogen atoms at the $C(4)$ and $C(5)$ positions which are crowded together closer than the sum of their van der Waals radii. At room temperature the molecule has $C_{2}$ symmetry, and these two hydrogens are on opposite sides of the central ring. In a temperature-dependent $x$-ray experiment by Matsumoto, ${ }^{17}$ the high- and low-temperature structures of phenanthrene were measured to show a very small change in unit cell dimensions, although it retained the monoclinic space group and $C_{2}$ factor group symmetry. Spielberg et al. ${ }^{18}$ performed temperature-dependent neutron scattering experiments and concluded that above the transition temperature, the two hydrogen atoms at positions $C(4)$ and $C(5)$ bend over to the same side of the central ring thereby possibly introducing another degree of freedom into the crystal.

Though it is quite apparent that this rearrangement of the hydrogens at $C(4)$ and $C(5)$ is responsible for the observed phase transition, the effect of this overcrowding has yet to be studied with regard to the phonon dispersion curves and the anisotropy of the potential in noncentrosymmetric lattices. Inspection of the phenanthrene crystal structure shows that both molecules in the unit cell lie essentially in the $a c$ plane and that the van der Waals forces, especially of the $\mathrm{H}-\mathrm{H}$ interactions, are very weak along the $b$ axis of the unit cell. The branch representing the high-frequency lattice mode appearing in our calculated dispersion curves is very flat in $c^{*}$ and very steep in $b^{*}$ because of the lack of sufficient repulsion normal to the $a c$ plane. This anisotropy, introduced by the strain caused by the intramolecular hydrogen interactions, is taken to be the main factor dictating the shape of the phenanthrene dispersion curves at low temperature.

\section{MOLECULAR MOTIONS}

Since all of the lattice vibrations of phenanthrene can be present in both the infrared and Raman spectra, the eigenvectors of the dynamical matrix are much more complicated than those of a crystal with centrosymmetric symmetry. Rather than undergoing motions that can be classified as pure translations or pure librations, the molecules in a noncentrosymmetric unit cell (here, the $C_{2}$ factor group) exhibit motions that combine librations with a change in the positions of molecules' centers of mass. Therefore, in describing these complex motions it is both convenient and practical to assign the modes as "mostly translational" or "mostly librational." Experimental confirmation of such an assignment can be qualitatively determined by measuring the intensities of each peak in both the infrared and Raman spectra. Since all modes can be present in each spectrum, the ratio of infrared and Raman intensities of a given mode can be examined to determine the degree of coupling between librational and translational lattice phonons.

Figures 1 and 2 show the motions of the molecules at

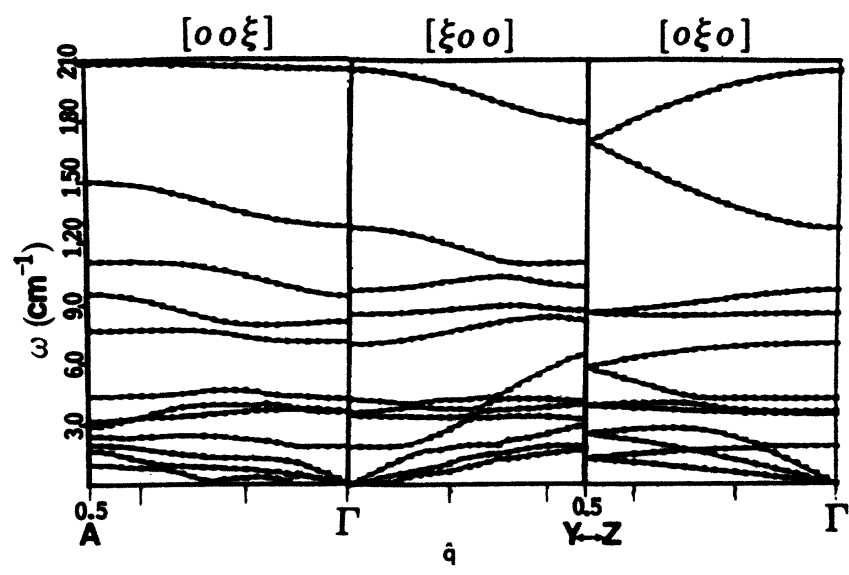

FIG. 1. Calculated phonon dispersion curves for phenanthrene. Evaluated from reduced wave vector $q=0$ to Brillouin zone boundary in $a^{*}, b^{*}$, and $c^{*}$ directions.

$q=0$ in the $\left(a, b, c^{*}\right)$ axis system. Of these nine optical phonons, only the two lowest-frequency vibrations show pure translational or pure rotational behavior. The remaining modes appear as translations and librations simultaneously, with rotations about axes parallel to $b$ being the most common at low frequencies and rotations about axes parallel to $c^{*}$ being present for higher energy lattice modes. These vectors show that the first three optical phonon modes involve librations of the phenenthrene molecule in the $a c$ plane. This analysis of the normal mode eigenvectors suggests that lattice vibrations of phenanthrene are more "two dimensional" at low temperatures than those of the other linear acene aromatic hydrocarbon crystals and could explain its unique phase transition as being driven by librations normal to the direction of low cohesive energy. Combined with the results of the density of states experimental data, the zero-wave-vector modes that have been calculated can be used to devise a viable mechanism for the phenanthrene phase transition which appears to differ significantly from others seen in molecular crystals.

\section{PROPOSED MECHANISM FOR THE PHENANTHRENE PHASE TRANSITION}

These lattice-dynamical calculations were carried out at a single temperature of $1 \mathrm{~K}$ and, as such, can in no way serve as the basis for a classical soft-mode phase transition study. Instead, we maintain that it is the symmetries of these complex coupled vibrations and their relationship to the lattice potential which are important in presenting this "molecular" picture of how Spielberg's proposed mechanism can be confirmed.

Though it is well accepted that a change in molecular geometry is the cause of the phenanthrene phase transition, a dynamic molecular mechanism has not been pro- 

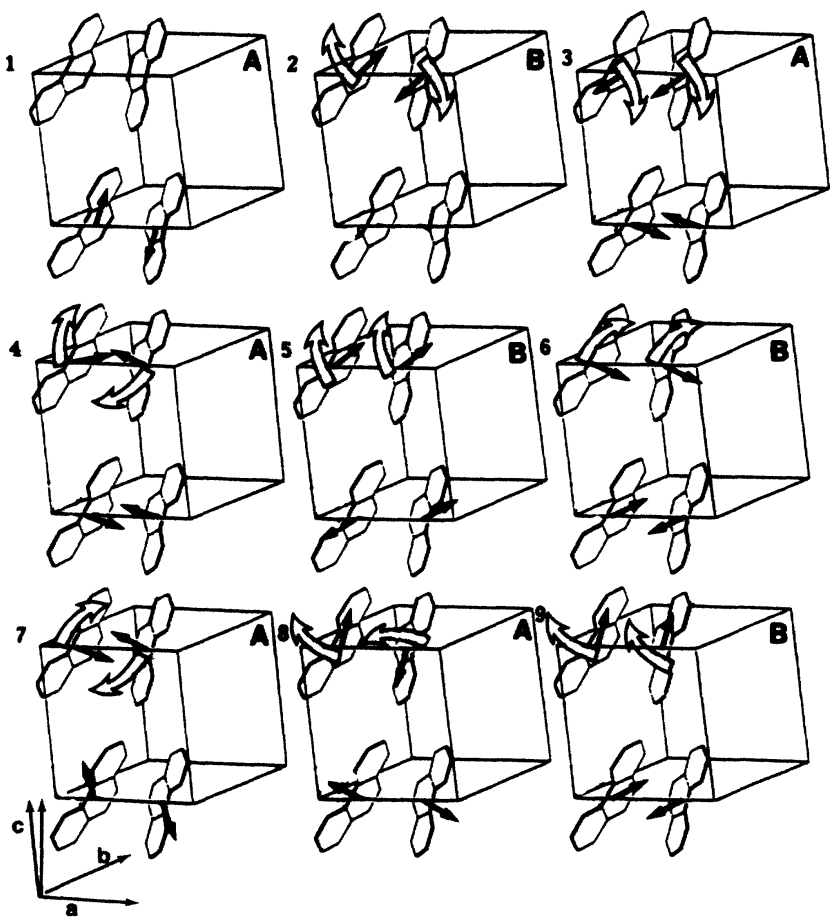

FIG. 2. Eigenvectors and symmetries describing the nine $(q=0)$ optical phonon modes in phenanthrene. Arrows on the upper pair of molecules in each unit cell represent polar vectors about which rotations occur. Translations are represented by arrows on the lower pairs of molecules. For example, mode 1 is a pure translation, and mode 2 is a pure rotation (libration). The remaining seven modes are coupled motions.

posed in light of the existing experimental results. The neutron data show a significant decrease in the density of the low-energy phonon states as the transition temperature is approached. This decrease in the density of lowenergy states, though it is to be expected, is more dramat- ic than that normally caused by thermal expansion.

The normalized eigenvectors in Table I show that the three lowest-energy optical modes (1-3) are composed of librations and translations that are restricted to occur entirely in the $a c$ plane. The protruding hydrogen atoms at $C(4)$ and $C(5)$ vibrate against the hydrogens in the adjacent molecules in such a manner as to retain enough attraction to keep all of these motions in the overcrowded ac plane (Fig. 3). Therefore, at low temperatures, the phonon states that are most likely to be populated will cause the phenanthrene molecules to vibrate in what can be regarded as a two-dimensional lattice.

At higher temperatures, the high-energy phonon states become more highly populated, and the eigenvectors of modes 8 and 9 show these librations occur about axes lying almost exclusively in the $a c$ plane, i.e., the $c^{*}$ axis. In this case, the protruding hydrogen atoms on each molecule twist away from those on the adjacent molecules and would then be free to flip over to the same side of the central ring (as shown in Fig. 4) to reduce steric hinderance. This is the observed conformation of the phenanthrene molecule above the $72^{\circ} \mathrm{C}$ transition temperature. Thus, this phase transition can be viewed as a change from essentially a two-dimensional lattice to a threedimensional lattice. From previous calculations and experiments, it has been shown that while the frequencies of the optical phonon modes in molecular crystals are temperature dependent, their symmetries remain unchanged unless a change in crystal symmetry occurs, i.e., there is a true structural phase transition. The symmetry of the phenanthrene lattice does not change during its phase transition, so the eigenvectors describing the molecular motions are representative of both the high- and lowtemperature phases. Therefore it is only the population of phonon states that changes over the transition temperature. Since the phonon density of states shows a significant decrease in available low-frequency vibrational levels at temperatures approaching $72^{\circ} \mathrm{C}$, it is reasonable

TABLE I. Zero wave-vector optical lattice modes and normalized eigenvectors.

\begin{tabular}{|c|c|c|c|c|c|c|c|c|c|}
\hline \multicolumn{10}{|c|}{ Frequencies $\left(\mathrm{cm}^{-1}\right)$} \\
\hline & Calc. & Expt. & Symmetry & $T_{x}$ & $T_{y}$ & $T_{z}$ & $R_{x}$ & $R_{y}$ & $R_{z}$ \\
\hline \multirow[t]{2}{*}{1} & 18.6 & 22 & $A$ & 0.438 & 0 & 0.897 & 0 & 0 & 0 \\
\hline & & & & -0.438 & 0 & -0.897 & 0 & 0 & 0 \\
\hline \multirow[t]{2}{*}{2} & 35.9 & 31 & $B$ & 0 & 0 & 0 & 0 & 1 & 0 \\
\hline & & & & 0 & 0 & 0 & 0 & -1 & 0 \\
\hline \multirow[t]{2}{*}{3} & 36.3 & 32 & $A$ & 0.871 & 0 & -0.306 & 0 & -0.381 & 0 \\
\hline & & & & -0.871 & 0 & 0.306 & 0 & -0.381 & 0 \\
\hline \multirow[t]{2}{*}{4} & 42.6 & 42 & $A$ & 0.775 & 0 & -0.455 & 0.244 & 0.360 & 0 \\
\hline & & & & -0.775 & 0 & 0.455 & -0.244 & 0.360 & 0 \\
\hline \multirow[t]{2}{*}{5} & 70.7 & 60 & $\boldsymbol{B}$ & 0 & -0.845 & 0 & 0.436 & 0 & 0.296 \\
\hline & & & & 0 & 0.845 & 0 & 0.436 & 0 & 0.296 \\
\hline \multirow[t]{2}{*}{6} & 80.9 & 84 & $B$ & 0 & 0.885 & 0 & 0.421 & 0 & -0.194 \\
\hline & & & & 0 & -0.885 & 0 & 0.421 & 0 & -0.194 \\
\hline \multirow[t]{2}{*}{7} & 93.5 & 107 & $\boldsymbol{A}$ & -0.377 & 0 & 0.299 & 0.788 & -0.377 & 0 \\
\hline & & & & 0.377 & 0 & -0.299 & -0.788 & -0.377 & 0 \\
\hline \multirow[t]{2}{*}{8} & 128.3 & 108 & $\boldsymbol{A}$ & -0.153 & 0 & 0 & 0 & 0 & 0.985 \\
\hline & & & & 0.153 & 0 & 0 & 0 & 0 & -0.985 \\
\hline \multirow[t]{2}{*}{9} & 205.6 & 141 & $\boldsymbol{B}$ & 0 & 0.681 & 0 & 0 & 0 & 0.729 \\
\hline & & & & 0 & -0.681 & 0 & 0 & 0 & 0.729 \\
\hline
\end{tabular}




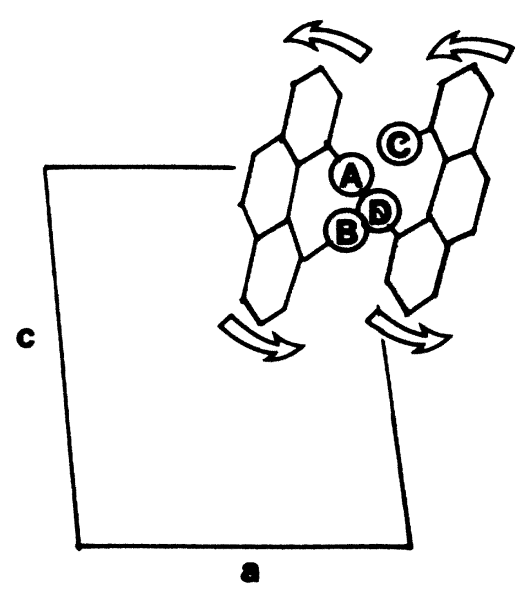

FIG. 3. Low-frequency vibrational mode showing the 4,5 hydrogen crowding among adjacent molecules. Approximate atom-atom distances between hydrogen atoms in Angstroms: $A D, 2.1 ; A C, 2.8 ; B D, 2.6 ; B C, 4.6$. The van der Waals radius of hydrogen in $1.2 \AA$.

to assume that above $72^{\circ} \mathrm{C}$ the lattice has an extra degree of vibrational freedom out of the $a c$ plane, as was suggested but not confirmed by Spielberg et al. In maintaining that this subtle phase transition can be likened to that of a lattice going from strongly two-dimensional interactions to those which are more three dimensional, it is interesting to note that the experimentally determined change in heat capacity over the $68-72^{\circ} \mathrm{C}$ temperature range is $0.8 \times 10^{-16} \mathrm{erg} / \mathrm{K}$. This value, which is very close to $k_{B} / 2$, would correspond to the change in heat capacity expected upon introduction of an additional degree of freedom into a system of harmonic oscillators.

The low-temperature crystal structure of phenanthrene reveals that the lattice is basically layered perpendicular to the $b$ axis. It is possible that such a phase change is most likely to be observed in similar crystals where the predominant atom-atom interactions between molecules occur essentially in only two dimensions at low temperatures.

\section{CONCLUSIONS}

The phenanthrene phase transition is problematic since it is more subtle in terms of energy and structure than is usually associated with order-disorder and displacive transitions. The usual study of phase transitions by following the temperature dependence of the frequency of the soft modes is possible for only a few molecular crystals, and phenanthrene is not among them. The approach taken to elucidate the mechanism was to study the lattice dynamics of phenanthrene with specific attention to the anisotropy of the crystal interactions and the nature of the molecular motions associated with the low-

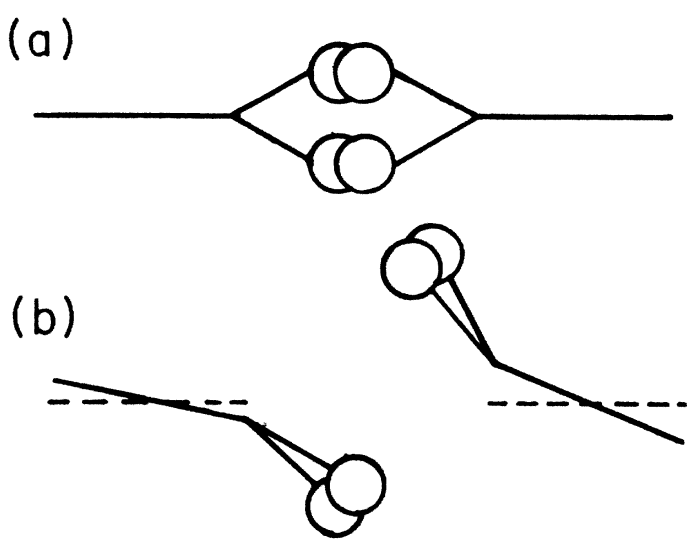

FIG. 4. Simple representation of phenanthrene phase transition mechanism. (a) The low-temperature (low-frequency vibrational mode) transoid configuration viewed along the short edge of the molecule. (b) The high-temperature (high-frequency vibrational mode) configuration where the molecules librate out of the $a c$ plane, and the crowded hydrogen atoms are forced into a cisoid arrangement to reduce intermolecular strain. Both views are essentially along the $c^{*}$ axis.

and high-frequency optical phonon $q=0$ eigenvectors.

The calculation has shown that there is a substantial difference in the nature of the motions associated with the low- and high-frequency lattice modes. These results can be joined with the observation from inelastic neutron scattering that shows a significant decrease in the density of low-frequency phonon states upon approach to the transition temperature. The picture which emerges is one wherein the low-frequency motions are confined to occur in the molecular layers perpendicular to the $b$ axis but, with increasing temperature, the high-frequency modes, which are largely out of the ac plane, show a significant increase in the density of states and confer a more three-dimensional character to the lattice.

The motions associated with the eigenmodes are consistent with the movement of the hydrogens from a lowtemperature transoid structure to one which is cisoid. When the layers begin to interact with one another, the repulsions are such that some stabilization can be expected when the hydrogens are on the same side of the molecular plane. This mechanism is unusual in that it implies that the phase transition is strongly associated with an internal mode. The heat capacity anomaly observed for the transition closely approximates the value of the change in heat capacity expected upon introducing an additional degree of freedom to a collection of harmonic oscillators, i.e., $k_{B} / 2$. This type of phase transition may occur in other crystals comprised of strained molecules like phenanthrene. It may also be speculated that it could occur in other less ordered phases such as liquid crystals and may serve as a model for changes in protein tertiary structure in enzymatic reactions. 
${ }^{1}$ R. Mason, Acta Cryst. 17, 547 (1964).

${ }^{2}$ K. M. White, R. C. Dye, and C. J. Eckhardt, Mol. Cryst. Liq. Cryst. 134, 265 (1986).

${ }^{3}$ G. M. Parkinson, M. J. George, S. Ramdas, J. O. Williams, and J. M. Thomas, J. Chem. Soc. Chem. Commun. 135, (1978). (1978).

${ }^{4} J$. Trotter, Acta Cryst. 16, 605 (1963).

${ }^{5}$ R. S. Bradley and T. G. Cleasby, J. Chem. Soc. 1690 (1953).

${ }^{6} \mathrm{~K}$. Ueberreiter and H. Orthmann, Z. Naturforschung. 5a, 101 (1950).

${ }^{7}$ S. Matsumoto and T. Tsukuda, Bull. Chem. Soc. Jpn. 38, 203 (1965).

${ }^{8}$ R. A. Arndt and A. C. Damask, J. Chem. Phys. 45, 4627 (1966).

${ }^{9}$ R. A. Arndt and A. C. Damask, J. Chem. Phys. 45, 755 (1966).

${ }^{10}$ A. I. Kitiagorodskii, Molecular Crystals and Molecules (Academic, New York, 1973).

${ }^{11}$ Thomas L. Starr and Donald E. Williams, Acta Cryst. A 33,
771 (1977)

${ }^{12}$ A. Chablo, D. W. J. Cruickshank, A. Hinchliffe, and R. W. Munn, Chem. Phys. Lett. 78, 424 (1981); R. W. Munn (private communication).

${ }^{13}$ I. A. Remizov, V. G. Podoprigora, A. N. Botvich, T. A. Kharitonova, and V. F. Shabanov, Chem. Phys. 92, 163 (1985).

${ }^{14} \mathrm{M}$. Born and K. Huang, Dynamical Theory of Crystal Lattices (Clarendon, Oxford, 1954).

${ }^{15}$ R. Righini, S. Califano, and S. H. Walmsley, Chem. Phys. 50, 113 (1980).

${ }^{16}$ S. L. Chaplot, G. S. Pawley, B. Dorner, V. K. Jindal, J. Kalus, and I. Natkaniec, Phys. Status Solidi B 110, 445 (1982).

${ }^{17}$ S. Matsumoto and T. Fukuda, Bull. Chem. Soc. Jpn. 40, 743 (1967).

${ }^{18}$ D. H. Spielberg, R. A. Arndt, A. C. Damask, and I. Lefkowitz, J. Chem. Phys. 54, 2597 (1971). 